\title{
Uma agenda de pesquisa para a Atenção Primária à Saúde no estado de São Paulo, Brasil: o estudo Elect
}

\author{
Gustavo Diniz Ferreira Gusso(h) \\ Janise Braga Barros Ferreira(i) \\ João Mazzoncini de Azevedo Marques ${ }^{(j)}$ \\ Luciana Cisoto Ribeiro(k) \\ Luciane Loures dos Santos(l) \\ Marcelo Marcos Piva Demarzo(m) \\ Paulo Celso Nogueira Fontão(n) \\ João Paulo Souza(o)
}

Eduardo Antônio de Sousa Orlandin (a)

Leonardo Moscovici(b)

Ana Carolina Arruda Franzon ${ }^{(c)}$

Afonso Dinis Costa Passos ${ }^{(\mathrm{d})}$

Amaury Lelis Dal Fabbro(e)

Elisabeth Meloni Vieira ${ }^{(f)}$

Fernando Bellissimo-Rodrigues ${ }^{(\mathrm{g})}$

Orlandin EAS, Moscovici L, Franzon ACA, Passos ADC, Dal Fabbro AL, Vieira EM, et al. A research agenda for Primary Health Care in the state of Sao Paulo, Brazil: the ELECT study. Interface (Botucatu). 2017; 21(61):349-61.

The consolidation of Primary Health Care (PHC) requires public policies based on scientific evidence. This paper presents the ELECT study, aimed to identify priority research themes for strengthening $\mathrm{PHC}$ in the state of Sao Paulo, Brazil. A list of the twenty main obstacles and ten priority research themes in $\mathrm{PHC}$ were obtained with participation of specialists and a focus group with users. The results point to problems and research issues related to organizational management, training of professionals and managers, professional development, creation of cooperation mechanisms between health teams and computerization of resources. It is expected to stimulate debate in the context of the $\mathrm{PHC}$ on the role of research prioritization, its obstacles and research propositions. It also aims to encourage the adoption of more participatory models of selection of research topics.

Keywords: Health research agenda. Health priorities agenda. Research prioritization. Primary Health Care. Primary Care.
A consolidação da Atenção Primária à Saúde (APS) requer políticas públicas embasadas por evidências científicas. Este artigo apresenta o estudo ELECT, cujo objetivo foi identificar temas prioritários de pesquisa para a fortalecimento da APS no estado de São Paulo, Brasil. Com a participação de especialistas e de um grupo focal com usuários, foi obtida uma lista com os vinte principais obstáculos, bem como dez temas de pesquisa prioritários, na APS. Os resultados apontam para problemas e temas de pesquisas relacionados à: organização da gestão, capacitação de profissionais e gestores, valorização profissional, criação de mecanismos de colaboração entre equipes de saúde e informatização dos recursos. Espera-se, assim, estimular o debate no contexto da APS sobre o papel da priorização de pesquisas, seus obstáculos e proposições de pesquisa. Almeja-se, também, estimular a adoção de modelos mais participativos de seleção de temas de pesquisa.

Palavras-chave: Agenda de Pesquisa em Saúde. Agenda de Prioridades em Saúde. Priorização de pesquisas. Atenção Primária à Saúde. Atenção Básica.

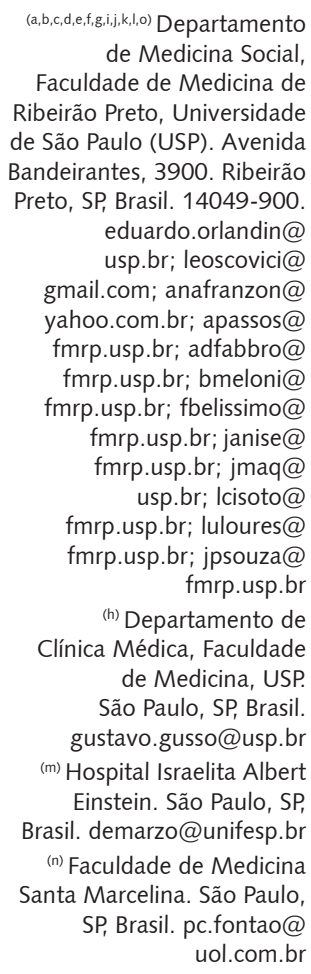

$(a, b, c, d, e, f, g, i, j, k, l, o)$ Departamento de Medicina Social, Faculdade de Medicina de Ribeirão Preto, Universidade de São Paulo (USP). Avenida Bandeirantes, 3900. Ribeirão Preto, SP, Brasil. 14049-900. eduardo.orlandin@ usp.br; leoscovici@ gmail.com; anafranzon@ yahoo.com.br; apassos@ fmrp.usp.br; adfabbro@ fmrp.usp.br; bmeloni@ fmrp.usp.br; fbelissimo@ fmrp.usp.br; janise@ fmrp.usp.br; jmaq@ usp.br; Icisoto@ fmrp.usp.br; luloures@ fmrp.usp.br; jpsouza@ fmrp.usp.br

(h) Departamento de

Clínica Médica, Faculdade de Medicina, USP. São Paulo, SP, Brasil. gustavo.gusso@usp.br

(m) Hospital Israelita Albert Einstein. São Paulo, SP, Brasil.demarzo@unifesp.br

(n) Faculdade de Medicina Santa Marcelina. São Paulo, SP, Brasil.pc.fontao@ uol.com.br 


\section{Introdução}

A Atenção Primária à Saúde (APS) é considerada o componente do sistema de saúde que serve de porta de entrada para o próprio sistema e é aquele que deve fornecer e coordenar cuidados abrangentes e centrados na pessoa e na comunidade (incluindo promoção de saúde, prevenção, tratamento e reabilitação) ${ }^{1-4}$. Seu funcionamento eficiente é um dos fundamentos para que dois dos principais objetivos de um sistema de saúde sejam atingidos: a otimização dos cuidados e a equidade do acesso ${ }^{5,6}$. Contudo, vários problemas têm dificultado o funcionamento pleno da APS em nosso meio e das estratégias a ela relacionadas ${ }^{7}$. Embora a superação de uma parcela substancial dos desafios da APS requeira vontade política e comprometimento para a formulação de políticas públicas informadas por evidências científicas e um planejamento de médio e longo prazo, a pesquisa e o desenvolvimento tecnológico podem contribuir para a formulação de soluções relevantes que podem acelerar o aperfeiçoamento dos serviços de saúde 8 .

Com uma população de mais de quarenta milhões de pessoas, São Paulo (SP), o estado mais populoso e com o maior PIB dentre todos os estados brasileiros, ainda busca melhorar sua atenção básica. Avanços importantes têm ocorrido na APS no estado, como o aumento do número de equipes de Saúde da Família9 . Entretanto, São Paulo ainda tem dificuldades para consolidar a Política Nacional de Atenção Básica. Dados do Departamento de Atenção Básica demonstram que, no município de Ribeirão Preto, por exemplo, com uma população de quase seiscentos e vinte mil habitantes, a cobertura de Estratégia de Saúde da Família (ESF) é de apenas 113.850 pessoas, o que equivale a pouco mais de $18 \%$. Mesmo os números do estado chamam atenção, com apenas pouco mais de 16 milhões de pessoas cobertas pela ESF, o que corresponde a, aproximadamente, $38 \%$ da população total $^{10}$. Outros entraves na APS do estado, muitos deles extrapoláveis para todo o Brasil, são: a falta de profissionais especializados em atenção básica, a cultura pelo modelo hospitalocêntrico, gestores sem capacitação adequada, etc. ${ }^{11,12}$.

Os exercícios de priorização de pesquisas podem auxiliar, possibilitando a elaboração e seleção de temas de pesquisa direcionados a superarem as barreiras existentes na APS ${ }^{13,14}$. A aplicação destes exercícios permite identificar obstáculos que, se abordados, produziriam maior potencial de transformação, bem como oportunidades que apresentam maior desempenho satisfatório ao serem aplicadas na realidade de cada população. A priorização de pesquisas é importante para possibilitar que o conhecimento científico atenda, de forma racional e equitativa, às demandas sociais ${ }^{15}$. Vale ressaltar que já existem agendas nacionais de pesquisa no Brasil, porém, até o momento, nenhuma específica voltada para APS ${ }^{16}$.

Este artigo apresenta o estudo ELECT - Estabelecendo Prioridades para o Desenvolvimento da Comunidade (Establishing Leads for Community Development), cujo objetivo geral foi definir uma agenda de prioridades em pesquisa com elevado potencial de fortalecer a APS e, assim, promover a saúde e o desenvolvimento social das comunidades. Os objetivos específicos do estudo foram: (i) identificar os principais problemas que, na opinião de especialistas e de integrantes da comunidade, constituam barreiras ao fortalecimento da APS; (ii) produzir propostas de pesquisa que possam solucionar os principais problemas da APS, e (iii) definir as perguntas prioritárias de pesquisa que auxiliem a promover a saúde das comunidades.

\section{Metodologia}

Trata-se de um estudo de métodos mistos (qualitativos e quantitativos), com dados produzidos por meio de consulta a um painel de especialistas e um grupo de usuários do SUS, executado em quatro fases (Figura 1). 


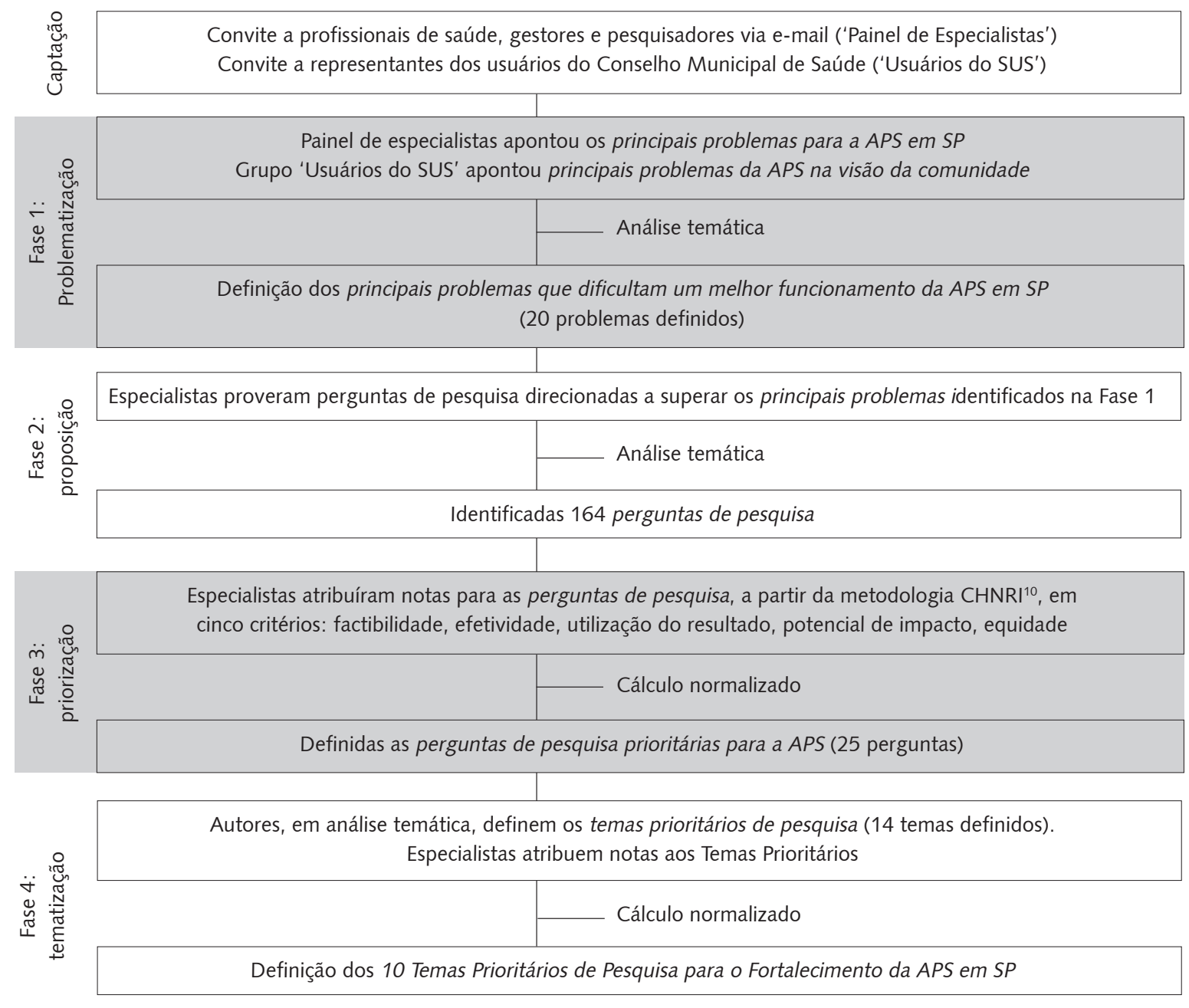

Figura 1. Fluxograma das quatro fases do estudo ELECT

(p) Associação Brasileira de Pós-Graduação em Saúde Coletiva. Rede de Pesquisa em Atenção Primária à

Saúde [Internet] [citado

2 Jan 2015]. Disponível em: http://www. rededepesquisaaps. org.br/

\section{Etapa preparatória: formação do painel de especialistas e do grupo de usuários}

Para a formação do painel de especialistas, definiram-se como critérios de elegibilidade: a) ser profissional de saúde, gestor ou pesquisador da área; b) atuar na APS no estado de SP; c) possuir e-mail público disponível nas fontes de pesquisa selecionadas para o estudo. Para isso, foram coletados os endereços de e-mail de potenciais especialistas a partir das seguintes fontes: (i) perfis públicos de profissionais cadastrados em páginas eletrônicas temáticas sobre APS (como a Rede de Pesquisa em Atenção Primária à Saúde)(p); (ii) páginas eletrônicas de departamentos de medicina preventiva e social de universidades públicas e privadas e de Departamentos Regionais de Saúde (DRS) de SP; (iii) consulta a 
artigos científicos na área de APS disponíveis na base de dados BIREME, utilizando as palavras-chave "Atenção Primária", "Saúde da Família" e "Medicina de Família"; (iv) lista de membros da Associação Paulista de Medicina de Família e Comunidade, mediante autorização prévia desta entidade. Aos endereços de e-mail obtidos, foram enviados os convites para participação no painel de especialistas do estudo.

A partir do convite via e-mail, os convidados deveriam acessar o link para uma plataforma online Google ${ }^{\circledR}$, que continha informações sobre a pesquisa; esclarecimentos para download do Termo de Consentimento Livre e Esclarecido; e botão para escolha de participar ou não do estudo. Ao não aceitar, o convidado visualizava uma mensagem de agradecimento, não sendo mais contatado para o estudo ELECT. Ao aceitar, o participante ingressava no painel de especialistas e tinha acesso liberado à fase um, em novo formulário online Google ${ }^{\circledR}$. Em todas as interações, os e-mails foram enviados em cópia oculta, de forma a preservar o anonimato e a não interação entre os participantes. Nas fases subsequentes, os participantes do painel receberam um e-mail convite para acessar o questionário.

Para a formação do grupo de usuários do SUS, o estudo foi apresentado, em reunião ordinária, aos conselheiros municipais de saúde de Ribeirão Preto, sendo aberto o convite aos participantes conforme os seguintes critérios de inclusão: ser usuário do SUS e membro do segmento dessa categoria no referido conselho. Após a formação do grupo, foi agendada uma atividade presencial.

\section{Fase um}

Em questionário online Google ${ }^{\circledR}$, a cada participante do painel de especialistas foi solicitado o preenchimento de duas seções. A primeira seção contemplava informações sociodemográficas: nome completo, e-mail de contato, profissão, número aproximado de habitantes no município em que tem atuação profissional predominante, Departamento Regional de Saúde paulista em que tem atuação predominante. Todos esses itens eram de resposta obrigatória.

Na segunda seção, deveria responder à pergunta: “Em sua opinião, quais são os três principais problemas que dificultam um melhor funcionamento da Atenção Primária à Saúde?". Dos três campos disponíveis para respostas, apenas um era de preenchimento obrigatório, e cada qual apresentava limite máximo de duzentos caracteres. Ao concluir, o participante era encaminhado a uma tela de agradecimento.

Concomitante às entrevistas online dos especialistas, o grupo dos usuários do SUS participaram de uma atividade presencial, coordenada por três membros da equipe (ACAF, EASO, JPS) deste projeto. Após a leitura do material explicativo, esclarecimento de dúvidas e assinatura do Termo de Consentimento Livre e Esclarecido pelos participantes, foi iniciada a atividade. A partir da técnica de Grupo Focal ${ }^{17}$, os participantes deveriam, a seu juízo, apontar e discutir problemas na APS e, ao final, definir em conjunto os dez mais relevantes.

Os problemas da APS apontados pelo painel de especialistas e usuários do SUS foram reorganizados por análise temática pelos autores do estudo, para: eliminar respostas duplicadas ou fora de âmbito, fundir conceitos semelhantes, e reestruturar respostas a fim de se atingir o nível de detalhe exigido pela pesquisa $^{18}$.

\section{Fase dois}

Cada participante do painel de especialistas, em questionário online Google ${ }^{\circledR}$, foi solicitado a identificar seu nome e e-mail (respostas obrigatórias). A seguir, eram apresentados os vinte principais problemas definidos na fase um, com a pergunta: "Baseado nos resultados da fase um, proponha uma pergunta de pesquisa que, em sua opinião, tem maior potencial de contribuir para a resolução dos problemas apontados e, por conseguinte, melhorar a Atenção Primária à Saúde" (preenchimento obrigatório). Ao final, o participante poderia também responder à pergunta opcional "Caso você considere algum problema que não foi apontado na lista acima, ou deseje promover uma outra ideia 
de pesquisa, use o espaço abaixo". Os dois campos de resposta continham um limite de trezentos caracteres cada.

As perguntas de pesquisa propostas pelos participantes foram reformuladas também por análise temática, de forma a serem utilizadas na fase três.

\section{Fase três}

Com base em análise de artigos do Child Health and Nutrition Research Initiative $(C H N R I)^{19}$, foram definidos, pelos pesquisadores deste estudo, os Cinco Critérios de Priorização de pesquisa a serem aplicados: factibilidade (a pergunta de pesquisa é capaz de ser respondida); efetividade da pesquisa (o conhecimento gerado é capaz de resultar ou produzir uma melhora na saúde das pessoas e das comunidades); utilização do resultado (o resultado da pesquisa será implementável, aceitável e terá um custo acessível); potencial de impacto (o resultado da pesquisa tem o potencial de melhorar significativamente a saúde das pessoas e das comunidades); equidade (a intervenção ou resultado terá capacidade de atingir os grupos que mais necessitam).

Definidos os critérios para priorização, uma nova consulta foi realizada ao painel de especialistas: cada participante, em questionário online Google ${ }^{\circledR}$, foi solicitado a identificar seu nome e e-mail (respostas obrigatórias); a seguir, era apresentado o texto:

\footnotetext{
“Na segunda fase, você e os demais participantes elaboraram perguntas de pesquisa que podem ajudar a superar os principais problemas identificados. Agora é o momento de priorizarmos as pesquisas - isto é, escolher quais as pesquisas que têm o maior potencial para ajudar a superar os problemas na Atenção Primária e, assim, contribuir para o desenvolvimento da comunidade. Para realizarmos a priorização, adaptamos o método CHNRI. Este método consiste em avaliar uma pergunta de pesquisa, e atribuir a ela uma nota global de 1 a 5 , baseado em Cinco Critérios de avaliação que buscam prever a aplicação da pesquisa no mundo real."
}

Nas páginas seguintes, o participante deveria ler cada uma das perguntas de pesquisa captadas na fase dois e atribuir uma nota de 1 (baixo) a 5 (alto) referente à capacidade de cada pergunta alcançar os cinco critérios - isto é, de ser factível, efetiva, utilizável, impactante e contribuir para a equidade em saúde -, tendo, à disposição, as definições dos critérios para consulta. Todas as perguntas de pesquisa eram de resposta opcional.

$\mathrm{Na}$ análise das respostas, para cada uma das perguntas de pesquisa, as notas dos participantes foram somadas e, em seguida, normalizadas (dessa forma, cada uma apresentou uma nota dentro do intervalo de 0,00 a 1,00). As perguntas de pesquisa do quartil superior (com nota igual ou superior a $0,75)$ foram selecionadas, constituindo as perguntas de pesquisa prioritárias.

\section{Fase quatro}

Na fase quatro, as perguntas de pesquisas prioritárias foram reorganizadas por análise temática, gerando temas prioritários de pesquisa. Em questionário online, Survey Monkey ${ }^{\circledR}$, cada participante do painel de especialistas foi solicitado a identificar seu nome e e-mail (respostas obrigatórias). A seguir, cada um dos temas prioritários deveria ser avaliado pelo participante, tendo como parâmetro os cinco critérios de priorização de pesquisas separadamente. Ou seja, o participante deveria responder a cinco páginas do questionário online, cada página considerando um dos cinco critérios, e no qual cada tema prioritário era avaliado em nota sim ou não, quanto à sua capacidade de alcançar o critério apontado. $\mathrm{Na}$ análise das respostas, foram somadas as notas dos participantes (sim:1; não:0) para cada tema prioritário, e as notas finais normalizadas. 


\section{Resultados}

\section{Etapa preparatória: formação do painel de especialistas e do grupo de usuários}

Um total de duzentos e cinquenta interessados contribuiu para a elaboração e priorização das opções de pesquisa. Houve participantes oriundos de todos os Departamentos Regionais de Saúde do estado, com a seguinte distribuição percentual: DRS 1-Grande São Paulo: 38,8\%; DRS 13-Ribeirão Preto: 22,4\%; DRS 7-Campinas: $10 \%$; demais DRS: participação entre 0,4\% e 5,6\%. O perfil quanto ao porte do município foi: mais de quinhentos mil habitantes: $58,4 \%$; entre cinquenta e quinhentos mil habitantes: $30,4 \%$; menos de cinquenta mil habitantes: 11,2\%. Quanto à profissão, a Figura 2 apresenta a distribuição percentual de cada categoria. Na captação dos representantes de usuários, sete conselheiros se voluntariaram para a atividade de Grupo Focal.

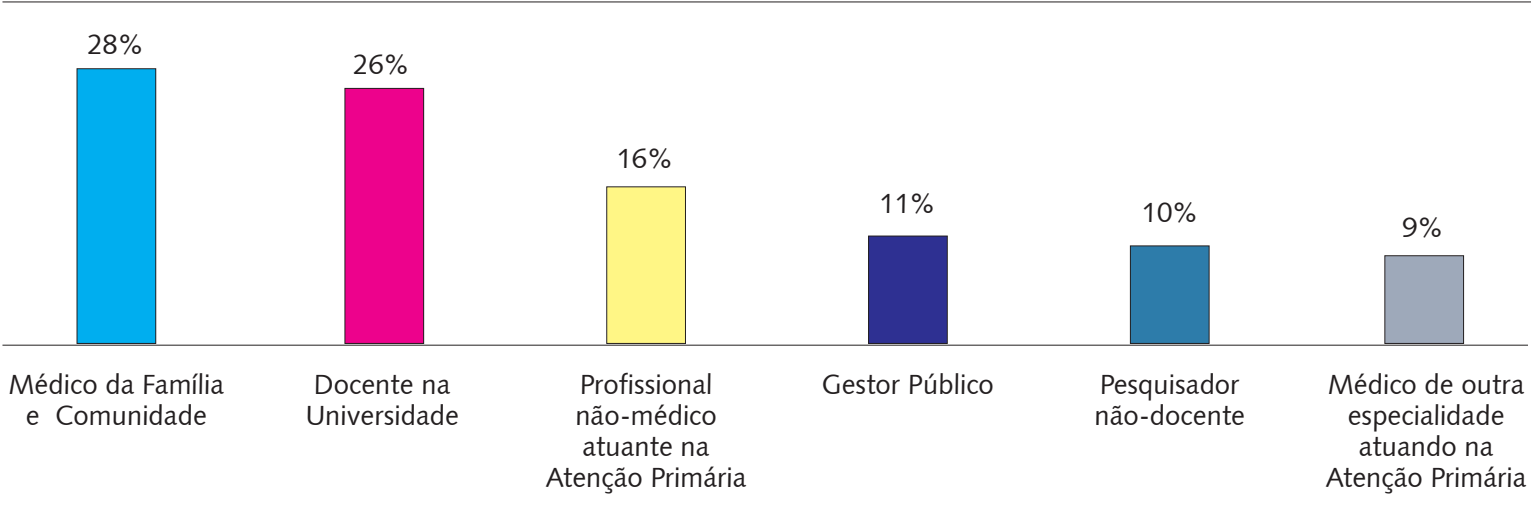

Figura 2. Distribuição percentual das categorias profissionais

\section{Fase um}

O painel de especialistas proveu setecentas e dez respostas, apontando o que seriam os principais problemas que dificultam um melhor funcionamento da APS. No exercício com os usuários do SUS, foram definidos dez principais problemas na APS na visão de usuários da comunidade. As respostas dos especialistas e dos usuários foram organizadas por análise temática e resultaram em vinte principais problemas que dificultam um melhor funcionamento da APS em SP (Quadro 1).

\section{Fase dois}

Houve 113 participações dentre os especialistas, obtendo-se igual número de perguntas de pesquisa principais (elaboradas com o foco de superar os vinte problemas selecionados na fase um), além de 29 perguntas de pesquisa opcionais (elaboradas com o foco superar problemas não apontados nos resultados da fase um), totalizando, assim, 142 perguntas de pesquisa com o objetivo de promover o fortalecimento da APS. Após análise temática - e considerando que diversas perguntas de pesquisa apresentadas pelos participantes incluíam, de fato, mais de uma pergunta - foram identificadas 164 perguntas de pesquisa. 
Quadro 1. Principais problemas e obstáculos para o fortalecimento da APS identificados na fase um

1 Falta de capacitação do profissional para APS, em especial para a Estratégia de Saúde da Família, além da falta de educação continuada

2 Falta de articulação, na rede de saúde, entre os níveis primário, secundário e terciário (dificuldades na referência e contra-referência), ou no matriciamento, dificultando a interação com especialistas focais

3 Carência ou erro de investimento do governo municipal e/ou estadual e/ou federal

4 Gestores sem capacitação em APS

5 Falta de valorização do profissional da APS: falta de reconhecimento, baixa motivação, baixo salário, condições de trabalho ruins, falta de plano de carreira

6 Carência de médicos especialistas em APS, especialmente médicos de família e comunidade, e carência de profissionais nãomédicos com perfil na APS

7 Visão curativo-medicamentosa e hospitalocêntrica em detrimento das ações de prevenção e promoção em saúde, por parte de profissionais, gestores e população

8 Desinteresse ou desconhecimento da população sobre APS e correta utilização de serviços

9 Deficiência de planejamento na APS

10 Unidades de saúde com infraestrutura inadequada (estrutura física precária, falta de equipamentos etc.)

11 Interferência negativa de políticos na saúde

12 Precariedade dos sistemas de informação (dificuldade de gerência de dados, excessivo preenchimento de documentos, falta de prontuário eletrônico)

13 Falhas, na graduação dos profissionais de saúde, quanto ao ensino de APS e sua relação com as comunidades

14 Falta de participação da sociedade para ajudar a melhorar o sistema de saúde - o controle social

15 Acolhimento de baixa qualidade: falta de humanização, falta de escuta, atenção ao idoso deficiente, atendimento desigual entre usuários

16 Baixa resolubilidade da APS

17 Falta de trabalho em equipe na APS

18 Alta rotatividade de profissionais na APS, principalmente médicos

19 Número de usuários cadastrados nas equipes de ESF é superior ao preconizado pelo Ministério da Saúde ou superior à capacidade resolutiva da equipe de APS

20 Falta de comprometimento de gestores com a APS

\section{Fase três}

Houve 91 participações de especialistas. Após seleção do quartil superior, foram identificadas 25 perguntas de pesquisa prioritárias para o fortalecimento da APS em SP (Quadro 2), as quais geraram 14 temas prioritários de pesquisa.

\section{Fase quatro}

Houve 93 participações de especialistas. Após cálculo das notas, foram obtidos os dez temas prioritários de pesquisa para o fortalecimento da Atenção Primária à Saúde no estado de São Paulo (Quadro 3). Maiores detalhes dos resultados do projeto ELECT estão disponíveis no site do estudo (http://elect.fmrp.usp.br/). 
Quadro 2. As 25 perguntas de pesquisa selecionadas na fase três; organização por temas prioritários.

\begin{tabular}{|c|c|}
\hline Pergunta de pesquisa (nota normalizada) & Temas prioritários \\
\hline $\begin{array}{l}\text { A implantação do prontuário eletrônico aumenta a efetividade da referência e contra- } \\
\text { referência dos usuários (incluindo a visualização e seguimento de resultados de exames de } \\
\text { imagem, exames laboratoriais)? }(1,00)\end{array}$ & \multirow{4}{*}{ 1. Prontuário eletrônico } \\
\hline $\begin{array}{l}\text { A implantação do prontuário eletrônico na atenção primaria à saúde e nos outros níveis de } \\
\text { atenção podem melhorar a continuidade do cuidado? }(0,95)\end{array}$ & \\
\hline $\begin{array}{l}\text { A implementação do prontuário eletrônico, permitiria a maior integração da rede, entre seus } \\
\text { diferentes níveis: primário, secundário e terciário? }(0,91)\end{array}$ & \\
\hline $\begin{array}{l}\text { A utilização de prontuários eletrônicos, que podem ser acessados em toda a rede de assistência } \\
\text { à saúde, pode melhorar a articulação entre os diversos níveis de saúde? }(0,76)\end{array}$ & \\
\hline $\begin{array}{l}\text { A informatização do SUS pode contribuir para a integração dos serviços de saúde em diferentes } \\
\text { níveis de complexidade? }(0,95)\end{array}$ & \multirow{3}{*}{$\begin{array}{l}\text { 2. Informatização dos } \\
\text { equipamentos de saúde }\end{array}$} \\
\hline $\begin{array}{l}\text { A informatização dos equipamentos de saúde, gerando informaçães sobre os usuários, dados } \\
\text { de produção, indicadores, controle da entrega dos remédios, poderia racionalizar a utilização } \\
\text { do sistema e ter mais eficiência evitando duplicidade de consultas, exames e gerenciando filas } \\
\text { de espera? }(0,91)\end{array}$ & \\
\hline A informatização do SUS pode otimizar a utilização dos recursos públicos na saúde? $(0,80)$ & \\
\hline $\begin{array}{l}\text { A definição de padrões ou protocolos básicos de processo de trabalho na atenção básica } \\
\text { como acolhimento com classificação de risco, matriciamento para especialidades, avaliação } \\
\text { de qualidade e ou remuneração por desempenho podem melhorar a resolubilidade da APS? } \\
(\mathbf{0}, \mathbf{8 7})\end{array}$ & \multirow[t]{2}{*}{$\begin{array}{l}\text { 3. Protocolos de atenção e } \\
\text { de regulação }\end{array}$} \\
\hline $\begin{array}{l}\text { A adoção de protocolos clínicos e regulatórios pode melhorar a resolubilidade da atenção } \\
\text { primária? }(0,82)\end{array}$ & \\
\hline $\begin{array}{l}\text { As reuniões entre equipes da estratégia de saúde da família para troca de experiências podem } \\
\text { melhorar a articulação das mesmas? }(0,78)\end{array}$ & \multirow{2}{*}{$\begin{array}{l}\text { 4. Reuniões entre equipes } \\
\text { da ESF }\end{array}$} \\
\hline $\begin{array}{l}\text { Encontros periódicos entre profissionais da APS e dos níveis secundário e terciário de atenção } \\
\text { podem favorecer a articulação entre os serviços, fortalecendo a rede de cuidados? }(0,87)\end{array}$ & \\
\hline As equipes multidisciplinares melhoram o resultado da Atenção Básica? $(0,85)$ & 5. Equipes multidisciplinares \\
\hline
\end{tabular}

continua

\section{Discussão}

Atualmente, não existe um consenso sobre qual o melhor método de priorização de pesquisas. A literatura sugere que, independentemente do método escolhido, este precisa ter: um contexto adequado; a utilização de uma abordagem compreensiva e detalhada; inclusividade (escolha dos grupos interessados com representatividade apropriada, incluindo o envolvimento da comunidade); uma busca adequada de informações para auxiliar o exercício; planejamento para posterior implementação; escolha adequada de critérios de priorização; escolha adequada do método para utilização das prioridades; avaliação das prioridades; e transparência ${ }^{14}$. 
Quadro 2. continuação

\begin{tabular}{|c|c|}
\hline Pergunta de pesquisa (nota normalizada) & Temas prioritários \\
\hline $\begin{array}{l}\text { A criação de um plano de carreira na atenção primária, com salário digno e dedicação integral } \\
\text { seria capaz de aumentar resolubilidade e satisfação dos usuários da ESF? }(0,87)\end{array}$ & $\begin{array}{l}\text { 6. Plano de carreira para os } \\
\text { profissionais da APS }\end{array}$ \\
\hline $\begin{array}{l}\text { Capacitações e especializações de gestores em APS podem melhorar a resolutividade na } \\
\text { Atenção Básica? }(0,76)\end{array}$ & $\begin{array}{l}\text { 7. Capacitação/ } \\
\text { especialização de gestores } \\
\text { em APS }\end{array}$ \\
\hline $\begin{array}{l}\text { Os estágios na APS (como parte dos cursos de graduação da área da saúde) podem favorecer a } \\
\text { integração do ensino, da pesquisa, e da assistência? }(0,85)\end{array}$ & \multirow{2}{*}{$\begin{array}{l}\text { 8. Integração do ensino e } \\
\text { pesquisa na Atenção Primária } \\
\text { à Saúde }\end{array}$} \\
\hline $\begin{array}{l}\text { Melhorar a articulação entre a universidade (ensino e pesquisa) e os serviços primários de } \\
\text { saúde contribui para aumentar a fixação dos recursos humanos na estratégia da Saúde da } \\
\text { Família? }(0,76)\end{array}$ & \\
\hline Quais são os principais determinantes da alta rotatividade de profissionais na APS? $(0,76)$ & \multirow{2}{*}{$\begin{array}{l}\text { 9. Estratégias para reduzir } \\
\text { a alta rotatividade de } \\
\text { profissionais da APS }\end{array}$} \\
\hline Quais intervenções podem reduzir a rotatividade de profissionais na APS? $(0,83)$ & \\
\hline $\begin{array}{l}\text { Quais são as estratégias efetivas para o aumento da motivação dos profissionais de saúde que } \\
\text { atuam na APS? }(0,79)\end{array}$ & $\begin{array}{l}\text { 10. Estratégias para } \\
\text { aumentar a motivação dos } \\
\text { profissionais de saúde que } \\
\text { atuam na APS }\end{array}$ \\
\hline $\begin{array}{l}\text { O uso de tecnologias da informação e comunicação para a melhor integração clínica entre } \\
\text { profissionais dos vários níveis de atenção pode impactar positivamente em indicadores de } \\
\text { saúde das populações assistidas? }(0,80)\end{array}$ & \multirow[t]{2}{*}{$\begin{array}{l}\text { 11. Tecnologias de } \\
\text { informação e comunicação }\end{array}$} \\
\hline $\begin{array}{l}\text { O uso da comunicação via web entre profissionais dos níveis primário, secundário e terciário } \\
\text { poderia facilitar a interação entre profissionais da atenção básica e especialistas focais? }(0,75)\end{array}$ & \\
\hline A educação permanente com os profissionais pode melhorar a resolubilidade da APS? $(0,76)$ & \multirow{2}{*}{$\begin{array}{l}\text { 12. Educação permanente } \\
\text { dos profissionais da APS }\end{array}$} \\
\hline $\begin{array}{l}\text { A educação permanente de profissionais de Atenção Primária à Saúde pode fortalecer o } \\
\text { modelo de atenção integral? }(0,75)\end{array}$ & \\
\hline $\begin{array}{l}\text { A ESF é mais efetiva do que o modelo tradicional de APS no tratamento de condições crônicas } \\
\text { como diabetes mellitus, hipertensão arterial sistêmica e asma brônquica? }(0,76)\end{array}$ & $\begin{array}{l}\text { 13. O modelo de atenção da } \\
\text { ESF para promoção da saúde } \\
\text { e prevenção e tratamento de } \\
\text { doenças }\end{array}$ \\
\hline $\begin{array}{l}\text { A contratação de profissionais de saúde de nível superior com especialização em saúde da } \\
\text { família melhora a qualidade e a resolubilidade do atendimento assim como organização do } \\
\text { serviço? }\end{array}$ & $\begin{array}{l}\text { 14. Especialização em saúde } \\
\text { da família }\end{array}$ \\
\hline
\end{tabular}

Quadro 3. Os 10 temas prioritários de pesquisa (fase quatro)

O modelo de atenção da ESF para promoção da saúde e prevenção e tratamento de doenças
Educação permanente dos profissionais da APS
Protocolos de atenção e de regulação
Equipes multidisciplinares
Capacitação/especialização de gestores em APS
Especialização em saúde da família
Integração do ensino e pesquisa na APS
Estratégias para aumentar a motivação dos profissionais de saúde que atuam na APS
Reuniões entre equipes da ESF
Informatização dos equipamentos de saúde


O projeto ELECT buscou atender a estes princípios norteadores, apesar de algumas limitações adiante descritas. O processo de priorização de pesquisas é de certa forma complexo, envolvendo: desde a identificação dos obstáculos principais que dificultam o melhor funcionamento de certa área, a criação de perguntas de pesquisa direcionadas a superar esses obstáculos, e, especialmente, a aplicação de mecanismos que permitam priorizar tais propostas.

Desta forma, o ELECT foi desenhado em fases para facilitar a compreensão do processo aos participantes. Entretanto, os mecanismos de priorização aplicados nas fases três e quatro - seleção de perguntas prioritárias de pesquisa e temas prioritários, respectivamente - podem ter causado certa confusão nos participantes.

A pesquisa obteve êxito no objetivo de captar a participação de diferentes grupos envolvidos na APS, abarcando profissionais de saúde, gestores, pesquisadores e, em especial, os representantes dos usuários. Este último grupo contribuiu para a identificação dos principais problemas na área. Foi gerada uma grande variedade de respostas - que podem ser úteis em pesquisas futuras - e diversos pontos de vista, evidenciando uma democratização da agenda de pesquisa.

Por outro lado, a inclusão de diferentes perfis de participantes, sobretudo em questionários à distância, exigiu esforços para oferecer instruções e obter respostas dentro do escopo do estudo. Entretanto, este esforço para instrução e treinamento dos participantes se traduziu por questionários mais longos e cansativos, reduzindo a adesão, o que pode ser observado neste estudo. Cabe refletir, dentro de cada proposta de priorização de pesquisa, qual seria a melhor estratégia no processo: captar um número grande de participantes (favorecendo a variedade de respostas), ou captar poucos participantes (permitindo um treinamento mais intensivo e com maior adesão).

Uma outra limitação deste estudo consistiu na heterogeneidade da participação de especialistas de acordo com as regiões de SP, e com o porte de municípios, apesar dos esforços investidos para captar um número grande e diversificado de especialistas em APS. Ademais, a categorização realizada não possibilitou a separação entre as profissões de saúde atuantes na APS para além dos médicos, como: enfermagem, fisioterapia, terapia ocupacional, odontologia, nutrição, assistência social e agentes comunitários de saúde. A categorização/classificação profissional de todos os participantes poderia ter auxiliado na identificação de problemas e soluções na APS na visão de cada setor/categoria, o que, eventualmente, poderia desencadear ações no âmbito de profissões específicas atuantes na APS.

A análise dos resultados da primeira fase (Quadro 1) permite observar que os principais problemas na APS envolvem a formação, estrutura e organização deste nível de atenção - e não problemas que envolvam, especificamente, a abordagem clínico-terapêutica do cuidado.

Conforme pode ser observado nos quadros 2 e 3, os achados deste trabalho sugerem que os principais desafios e propostas de soluções envolvem temas estruturantes da APS: organização da gestão, capacitação de profissionais e gestores, valorização profissional, criação de mecanismos de colaboração entre equipes e informatização dos recursos. Ainda que os temas identificados pelo grupo consultivo do ELECT sugiram a grande importância das questões organizacionais ou de aplicação de ações já reconhecidas (e que, frequentemente, demandam comprometimento dos tomadores de decisão e vontade política), a pesquisa científica - particularmente a pesquisa de implementação pode contribuir para o aperfeiçoamento dos serviços e auxiliar o desenvolvimento de estratégias que favoreçam a implantação sustentável de soluções para problemas que afetam a APS 11,20,21.

A participação do grupo de usuários do SUS ocorreu em número adequado (a metodologia recomenda de quatro a oito participantes) ${ }^{17}$. A participação foi limitada a um município do estado de SP, pois dadas as limitações orçamentárias do ELECT, foi considerada inviável a captação de representantes de usuários de demais regiões do estado. Foi possível observar, nos representantes, grande experiência e interesse na fiscalização e desenvolvimento do SUS.

Em relação à originalidade, o estudo ELECT é o primeiro no Brasil a identificar prioridades de pesquisa na APS utilizando a metodologia CHNRI, e o primeiro, no mundo, a envolver a participação de usuários da comunidade nesta metodologia. 


\section{Próximos passos}

Mais do que uma lista com opções prioritárias de pesquisa relacionadas à APS, o grupo de pesquisadores e interessados em torno desta iniciativa pretende estimular a criação de uma agenda de pesquisa voltada para a solução dos principais problemas enfrentados pelos serviços de APS no estado. Este grupo espera que os resultados do projeto ELECT possam subsidiar exercícios futuros, impulsionando mudanças positivas que fortaleçam a APS brasileira, a reorganização da rede de atenção do Sistema Único de Saúde e o cuidado à saúde dos indivíduos e das comunidades. Também existe a expectativa de contribuir para ampliar a participação e controle social na pesquisa e desenvolvimento tecnológico, e superar o modelo tradicional de pesquisa, que tem se mostrado insuficiente para combater a "Desigualdade 90/10", onde 90\% das pesquisas são realizadas sobre condições que afetam apenas $10 \%$ da população mundial ${ }^{22}$. Cada vez mais, nos últimos anos, as políticas informadas por evidências são requisitadas globalmente, assumindo um papel fundamental no complexo universo dos sistemas de saúde ${ }^{23,24}$. A adoção de modelos mais participativos que atendam não apenas ao mérito acadêmico, mas, também, e, sobretudo, à relevância social, pode fortalecer a interação entre pesquisa, assistência e extensão, e contribuir, efetivamente, para a melhora da saúde de nossa população.

\section{Colaboradores}

Eduardo Antônio de Sousa Orlandin, Leonardo Moscovici, Ana Carolina Arruda Franzon e João Paulo Souza participaram: da elaboração do projeto, da execução ao longo de toda a pesquisa, da discussão dos resultados, da revisão e da aprovação da versão final do trabalho. Afonso Dinis Costa Passos, Amaury Lelis Dal Fabbro, Elisabeth Meloni Vieira, Fernando Belissimo Rodrigues, Janise Braga Barros Ferreira, João Mazzoncini de Azevedo Marques, Luciana Cisoto Ribeiro e Luciane Loures dos Santos participaram: da elaboração do projeto, da discussão dos resultados e da revisão e da aprovação da versão final do trabalho. Gustavo Diniz Ferreira Gusso, Marcelo Marcos Piva Demarzo e Paulo Celso Nogueira Fontão trabalharam na discussão dos resultados e revisão e aprovação da versão final do trabalho.

\section{Agradecimentos}

Agradecemos a todos os 257 profissionais de saúde, gestores, pesquisadores e representantes dos usuários do SUS, que participaram nas diversas fases do ELECT SP.

\section{Referências}

1. Starfield B. Is primary care essential? Lancet. 1994; 344(8930):1129-33.

2. Ministério da Saúde (BR). As Cartas da Promoção da Saúde. Brasília (DF): Secretaria de Políticas de Saúde; 2002.

3. Mendes EV. As redes de atenção à saúde. 2a ed. Brasília (DF): Organização PanAmericana da Saúde; 2011. 
4. Kringos DS, Boerma WGW, Hutchinson A, Van der Zee J, Peter P. The breadth of primary care: a systematic literature review of its core dimensions. BMC Health Serv Res [Internet]. 2010 [citado 4 Jan 2016]; 10(1):65. Disponível em: http://www.biomedcentral. com/1472-6963/10/65

5. World Health Organization. The World Health Report 2008: primary health care now more than ever [Internet]. Geneva: World Health Organization; 2007 [citado 10 Jan 2016]. Disponível em: http://www.who.int/whr/2008/en/

6. Mendes EV. O cuidado das condições crônicas na atenção primária à saúde: o imperativo da consolidação da estratégia da saúde da família. Brasília (DF): Organização Pan-Americana da Saúde; 2012.

7. Gillam S. Is the declaration of Alma Ata still relevant to primary health care? BMJ. 2008; 336(7643):536-8.

8. Lau R, Stevenson F, Ong BN, Dziedzic K, Treweek S, Eldridge S, et al. Achieving change in primary care - causes of the evidence to practice gap: systematic reviews of reviews. Implement Sci [Internet]. 2016 [citado 25 Mar 2016]; 11: 40. Disponível em: https:// implementationscience.biomedcentral.com/articles/10.1186/s13012-016-0396-4

9. Sala A, Mendes JDV. Perfil de indicadores da atenção primária à saúde no estado de São Paulo: retrospectiva de 10 anos. Saude Soc [Internet]. 2011 [citado 4 Jan 2016]; 20(4):912-26. Disponível em: http://www.scielo.br/scielo.php?script=sci_ arttext\&pid=S0104-12902011000400009\&lng=en

10. Secretaria da Saúde do Estado de São Paulo. Departamento de Atenção Básica [Internet]. Dados entre janeiro/2014 e janeiro/2015 [citado 1 Ago 2015]. Disponível em: http://dab.saude.gov.br/dab/historico_cobertura_sf/historico_cobertura_sf_relatorio.php

11. Paim J, Travassos C, Almeida C, Bahia L, Macinko J. The Brazilian health-system: history, advances, and challenges. Lancet. 2011; 377(9779):1778-97.

12. Arantes $L$, Shimizu HE, Merchán-Hamann E. The benefits and challenges of the Family Health Strategy in Brazilian Primary Health care: a literature review. Cienc Saude Colet. 2016; 21(5):1499-510.

13. Pellegrini-Filho A. Pesquisa em saúde, política de saúde e eqüidade na América Latina. Cienc Saude Colet. 2004; 9(2):339-50.

14. Viergever FR, Olifson S, Ghaffar A, Terry FR. A checklist for health research priority setting: nine common themes of good practice. Health Res Policy Syst [Internet]. 2010 [citado 4 Jan 2016]; 8(1):36. Disponível em: https://www.k4health.org/toolkits/researchutilization/checklist-health-research-priority-setting-nine-common-themes-good

15. Santos LMP, Souza LEPF. Serruya SJ, Guimarães RFN. O papel da pesquisa na consolidação do Sistema Único de Saúde (SUS). Cad Saude Publica. 2010; 26(9):1666-7.

16. Ministério da Saúde (BR). Secretaria de Ciência, Tecnologia e Insumos Estratégicos. Departamento de Ciência e Tecnologia. Agenda nacional de prioridades de pesquisa em saúde. 2a ed. Brasília (DF): Ministério da Saúde; 2008.

17. Kitzinger J. Introducing focus groups. BMJ. 1995; 311(7000):299-302.

18. Boyatzis RE. Transforming qualitative information: thematic analysis and code development. Thousand Oak: Sage Publications; 1998.

19. Rudan I, Gibson J, Kapiriri L, Lansang MA, Hyder AA, Lawn J, et al. Setting priorities in global child health research investments: assessment of principles and practice. Croat Med J. 2008; 48(5):595-604.

20. Doubova SV, Guanais FC, Pérez-Cuevas R, Canning D, Macinko J, Reich MR. Attributes of patient-centered primary care associated with the public perception of good healthcare quality in Brazil, Colombia, Mexico and El Salvador. Health Policy Plan. 2016; 31(7):834-43. 
21. Lemos N. Sugestões para gestores municipais agirem ativamente no processo de implementação da atenção primária em saúde. In: Organização Pan-Americana da Saúde, organizador. Inovando o papel da atenção primária nas redes de atenção à saúde: resultados do laboratório de inovações em quatro capitais brasileiras. Brasília (DF): Opas, Ministério da Saúde, Conass, Conasems; 2011. 117-128.

22. Global Forum for Health Research. The 10/90 Report on Health Research 2003-2004 [Internet]. Geneve: GFHR; 2004 [citado 10 Jan 2016]. Disponível em: http://apps.who. int/iris/handle/10665/44386

23. Langlois EV, Becerril Montekio V, Young T, Song K, Alcalde-Rabanal J, Tran N. Enhancing evidence informed policymaking in complex health systems: lessons from multi-site collaborative approaches. Health Res Policy Syst [Internet]. 2016 [citado 25 Mar 2016]; 14(2016):20. Disponível em: http://health-policy-systems.biomedcentral.com/ articles/10.1186/s12961-016-0089-0

24. El-Jardali F, Lavis J, Moat K, Pantoja T, Ataya N. Capturing lessons learned from evidence-to-policy initiatives through structured reflection. Health Res Policy Syst [Internet]. 2014 [citado 1 Jan 2016]; 12(1):2. Disponível em: https://health-policysystems.biomedcentral.com/articles/10.1186/1478-4505-12-2.

Orlandin EAS, Moscovici L, Franzon ACA, Passos ADC, Dal Fabbro AL, Vieira EM, et al. Una agenda de investigación para la Atención Primaria de Salud en el estado de Sao Paulo, Brasil: el estudio ELECT. Interface (Botucatu). 2017; 21(61):349-61.

La solidificación de la Atención Primaria de Salud (APS) requiere políticas públicas con base en evidencias científicas. Este artículo presenta el estudio ELECT, cuyo objetivo fue identificar temas prioritarios de investigación para el fortalecimiento de la APS en el estado de São Paulo. Con la participación de especialistas y de un grupo de opinión formado por usuarios, se obtuvo una lista con los veinte principales obstáculos, así como diez temas de investigación prioritarios en la APS. Los resultados señalan problemas y temas de investigación relacionados a la organización de la gestión, la capacitación de profesionales y gestores, la valorización profesional, la creación de mecanismos de colaboración entre equipos de salud e informatización de los recursos. Se espera por lo tanto incentivar el debate en el contexto de la APS sobre el papel de la priorización de investigaciones, sus obstáculos y propuestas de investigación. Se anhela también incentivar la adopción de modelos más participativos de selección de temas de investigación.

Palabras clave: Agenda de investigación en salud. Agenda de prioridades en salud. Priorización de la investigación. Atención Primaria de Salud. Atención básica. 
